Annuaire suisse de politique de développement

25-2 | 2006

Paix et sécurité : les défis lancés à la coopération internationale

\title{
Politique de développement dans les Balkans occidentaux : points de vue du terrain
}

\section{Andreas Ernst}

\section{(2) OpenEdition}

\section{Journals}

Édition électronique

URL : http://journals.openedition.org/aspd/277

DOI : $10.4000 /$ aspd. 277

ISSN : 1663-9669

Éditeur

Institut de hautes études internationales et du développement

Édition imprimée

Date de publication : 1 octobre 2006

Pagination : 207-221

ISBN : 2-88247-064-9

ISSN : 1660-5934

Référence électronique

Andreas Ernst, "Politique de développement dans les Balkans occidentaux : points de vue du terrain », Annuaire suisse de politique de développement [En ligne], 25-2 | 2006, mis en ligne le 01 octobre 2006, consulté le 07 septembre 2020. URL : http://journals.openedition.org/aspd/277 ; DOI : https://doi.org/ $10.4000 /$ aspd. 277 


\title{
Politique de développement dans les Balkans occidentaux: points de vue du terrain
}

\author{
Andreas Ernst*
}

\section{Introduction}

En se rendant de Belgrade à Skopje par l'autoroute en été ou en feuilletant l'annuaire téléphonique d'une grande ville suisse, on pourrait penser que les Balkans occidentaux ${ }^{1}$ font partie de l'étranger proche de la Suisse. En effet, des milliers de ressortissants d'ex-Yougoslavie, d'origine albanaise, serbe ou macédonienne, vont passer leurs vacances à Pristina, à Belgrade ou à Skopje avec leur voiture immatriculée en Suisse. Le reste de l'année, ils vivent et travaillent dans notre pays ${ }^{2}$. Cependant, contrairement à ce que pourrait suggérer l'expression postsoviétique «étranger proche», les Balkans occidentaux ne sont pas une zone d'influence exclusive de la Suisse. Il n'en reste pas moins que les relations personnelles étroites entre cette région et notre pays influent sensiblement sur la politique étrangère de la Suisse. En effet, notre pays abrite une diaspora exyougoslave de 300'000 personnes (400'000 selon certains). Selon la situation politique, elle fait l'effet soit d'un aimant, attirant d'autres émigrés des Balkans occidentaux, soit d'un exportateur de prospérité: chaque année, les migrants transfèrent quelque 240 millions de francs suisses chez eux. Dans un document stratégique adopté au printemps 2002, le Conseil fédéral a dès lors fait de l'Europe du Sud-Est la région prioritaire de sa politique étrangère. En conséquence, c'est à cette région qu'est destinée la majeure partie de l'aide aux pays de l'Est ${ }^{3}$.

Les considérations ci-après sur la politique suisse à l'égard des Balkans occidentaux s'articulent en cinq parties. La première explique pourquoi la Suisse réserve une grande place à cette région sise à la périphérie de l'Union européenne (UE).

* Correspondant de la Neue Zürcher Zeitung (NZZ) et de la NNZ am Sontag pour l'Europe du Sud-Est, Skopje, République de Macédoine.

1 Les Balkans occidentaux désignent les pays issus de l'ex-Yougoslavie, sans la Slovénie, mais avec l'Albanie. Les voici: Croatie, Serbie (y compris le Kosovo administré par l'oNU), Monténégro, Bosnie-Herzégovine, Macédoine et Albanie. Bien que l'expression «Balkans occidentaux» soit sur toutes les lèvres depuis quelques années, on ne parle que rarement des dénominateurs communs des pays de la région. Sur le plan structurel, ils ont en commun les caractéristiques suivantes : insuffisance des capacités administratives de l'autorité centrale, faiblesse de l'appareil judiciaire et clientélisme des élites politiques; économies en transition avec un taux d'investissement faible et un chômage élevé; sociétés marquées par des divisions ethniques, avec une opinion publique tout aussi divisée.

2 On les trouve donc dans nos annuaires téléphoniques, de « $\mathrm{A}$ » comme Ademi à « $\mathrm{Z}$ » comme Zivkovic.

3 Stratégie de politique extérieure de la Suisse pour l'Europe du Sud-Est, adoptée le 24 avril 2002, p. III. La West Balkan Strategy 2007 to 2010 de la Direction du développement et de la coopération (DDC) ne s'en éloigne pas fondamentalement. L'objet principal - prévenir la migration - est simplement masqué derrière des formules telles que «coopération internationale» et «accroissement de l'efficience» (SDC, West Balkan Strategy 2007 to 2010, avant-projet, document de travail, 6 avril 2006, p. 9. 
Il en ressort que l'attention de la Suisse est essentiellement motivée par sa volonté d'empêcher l'immigration en provenance de cette zone. La deuxième partie montre à quel point la Suisse calque sa politique de développement sur celle de l'Union européenne. La stratégie européenne d'élargissement (qui ne va pas sans contestations internes) offre une chance unique d'instaurer une paix et un développement durables dans les Balkans occidentaux. Elle sera couronnée de succès lorsque les pays en transition deviendront des Etats membres de l'UE, c'est-à-dire lorsque le processus de création d'Etats membres sera achevé. La troisième partie considère les activités de certains bureaux de coopération de la Direction du développement et de la coopération (DDC) pour illustrer les tendances de cette coopération. Il en ressort que les opérations visent clairement à favoriser la mise en place d'Etats de droit pleinement opérationnels. La quatrième partie examine la composante militaire de l'engagement suisse. Enfin, la cinquième partie s'interroge sur la cohérence de la politique des divers acteurs suisses. La planification et la mise en œuvre des activités du Département fédéral des affaires étrangères (DFAE) et du Département fédéral de la défense, de la protection de la population et des sports (DDPS) sont étroitement coordonnées. Il reste à savoir comment la prise de position du DFAE - qui s'est déclaré en faveur de l'indépendance du Kosovo - influence le travail quotidien des coopérants suisses sur place. Dans ce cadre, nous examinerons l'harmonisation du travail à la centrale et sur le terrain.

Principal objectif de la politique suisse dans les Balkans: prévenir l'immigration

La politique suisse dans les Balkans est principalement motivée par un objectif défensif: prévenir autant que possible tout nouveau mouvement migratoire issu de la région. Au-delà des déclarations médiatiques, cet objectif transparaît clairement dans les documents stratégiques de la politique étrangère. Si la Suisse n'est pas présente dans les Balkans, les Balkans viendront en Suisse! Voilà le genre de raisonnements schématiques avancés pour expliquer aux contribuables le montant des dépenses consenties pour promouvoir la sécurité et le développement dans les Balkans occidentaux: plus de 1,5 milliard de francs depuis 1995.

Ayant connu divers afflux de réfugiés, partis pendant les crises des Balkans et entrés sur son territoire avec l'aide de leurs compatriotes résidant ici, la Suisse entend désormais empêcher l'immigration en provenance de cette région ${ }^{4}$. Parmi les «intérêts spécifiques de la Suisse», le document stratégique mentionne la «prévention de flux massifs de réfugiés et de migrations» ainsi que la

4 L'immigration a littéralement explosé pendant la guerre de Bosnie, entre 1992 et 1995, et surtout pendant le conflit du Kosovo, entre 1998 et 1999: 70\% de tous les réfugiés venaient alors d'Europe du Sud-Est. L'immigration en provenance des Balkans relève d'une tradition qui remonte aux années 1970. Aujourd'hui, les travailleurs migrants de la région ne sont pas admis en Suisse, mais nombre de personnes, des jeunes en particulier, n'hésitent pas à essayer d'entrer illégalement dans notre pays. Bien que leurs chances d'admission soient pour ainsi dire nulles, la majorité des requérants d'asile proviennent toujours des Balkans occidentaux (il s'agit principalement de minorités du Kosovo et de Serbie). Mais les migrants des Balkans seront bientôt remplacés par les requérants d'asile venus d'Afrique (subsaharienne), dont la proportion parmi les demandeurs d'asile a presque doublé ces dix dernières années. 
«lutte contre l'extrémisme politique et la criminalité organisée». L'intérêt de la Suisse à contribuer au développement de l'économie et des pôles d'investissement des Balkans occidentaux, dont les 20 millions d'habitants représentent un marché potentiel privilégié pour les produits suisses, ne vient qu'en deuxième position. Nombre d'observateurs se déclarent d'ailleurs surpris par la réserve des investisseurs suisses, au vu de l'enthousiasme qui anime les Autrichiens, les Allemands et les Italiens. On l'explique en général par la (trop) grande prudence typiquement helvétique. Dans le domaine culturel, les échanges restent également modestes 5 . En Suisse, la région est perçue comme étrangère, mais peu attrayante car elle n'est pas exotique. L'image que les médias en ont donnée dans les années 1990 a été marquée par un nationalisme exacerbé, une violence à fleur de peau et la misère économique. Et la mauvaise réputation des émigrants ${ }^{6}$ ne fait que perpétuer ces clichés ${ }^{7}$.

La Suisse partage avec l'Union européenne l'idée que la prévention de l'immigration passe par le développement et aligne largement sa politique sur celle de l'UE. L'objectif consiste à améliorer au plus vite la situation dans les pays balkaniques pour leur permettre d'adhérer à l'UE. En participant à cet effort, la Suisse sait néanmoins, comme en témoigne le débat actuel sur la contribution au Fonds de cohésion de l'UE, que ses obligations financières ne s'arrêteront pas là̀. La Direction du développement et de la coopération (DDC), certainement le principal acteur suisse dans la région, élabore actuellement une stratégie 2007 à 2010 pour les Balkans occidentaux 9 . Ce document qualifie la situation initiale de la région de très disparate. Il constate que toute menace immédiate de conflit militaire est écartée (sans que l'on puisse exclure de possibles flambées de violence) et que la région a fait de nets progrès en matière de stabilisation et de réconciliation, mais que le passage à l'économie de marché prend du temps. En effet, malgré des taux de croissance élevés, le niveau de vie demeure inférieur à celui mesuré au début des années 1990, et n'atteint que $25 \%$ environ de la moyenne de l'UE. Par ailleurs, la croissance aurait surtout été alimentée par le rapatriement des salaires de la diaspora et par l'aide étrangère, alors que les investissements directs étrangers seraient modestes. En conséquence, le chômage reste élevé. L'aide internationale aurait permis de mettre en place les premières structures politiques et administratives et lancé la réhabilitation des infrastructures.

5 La Fondation Pro Helvetia, organisme de droit public, réalise dans les Balkans occidentaux (ainsi qu'en Ukraine) un programme qui ne vise pas à multiplier les échanges culturels avec la Suisse, mais à promouvoir les initiatives culturelles locales, car de telles initiatives devraient contribuer à renforcer une société civile critique. Le programme est financé par la DDC (voir plus bas la section «Politique autonome de la Suisse dans une zone d'influence européenne»).

6 On les appelle les «Jugos» en Suisse alémanique et les «Youyous» en Suisse romande.

7 L'attitude suisse à l'égard des Balkans occidentaux est comparable, à plus d'un titre, à celle des citadins français à l'égard des banlieues qui s'enflamment. On ne prend conscience de leur existence que lorsqu'il s'y passe quelque chose. Peu intéressante en soi, cette «zone à problèmes » gagne en importance lorsque l'on se rend compte que son développement approprié sert utilement ses propres intérêts.

8 Cf. «Begehren um Kohäsionsbeiträge vor der Tür. Ausdehnung der bilateralen Veträge bei EU-Erweiterung im Osten », Neue Zürcher Zeitung (NZZ), n 113, 17. Mai 2006, p. 34.

9 Pour la DDC, les Balkans occidentaux comprennent la Serbie (y compris le Kosovo, administré par l'ONU), le Monténégro, la Bosnie-Herzégovine, la Macédoine et l'Albanie. Contrairement à la définition retenue par l'UE, la Croatie n'en fait pas partie, car la DDC n'y mène aucun projet de coopération. Cf. Swiss Agency for Development and Cooperation (SDC), West Balkan Strategy 2007 to 2010, op. cit. 
Enfin, le document constate que le processus de reconstruction soutenu par l'aide étrangère touche à sa fin.

On pourrait sans doute remettre en question certains détails de cette appréciation. Le tableau de la relance économique est en particulier trop sombre, notamment si l'on considère la «ruée vers l'or» que connaît aujourd'hui le marché financier serbe ou l'accroissement des exportations, dont la valeur a augmenté de $30 \%$ en moyenne ces dernières années ${ }^{10}$. Globalement, l'analyse reste toutefois pertinente: dans les divers pays des Balkans, la relance économique et la stabilisation n'en sont qu'à leurs débuts et leur succès est loin d'être garanti. Ce dont la région a besoin aujourd'hui - et, là encore, le document fait mouche -, c'est d'une réorientation de l'aide. «Celle-ci devrait en effet atténuer l'effort de réhabilitation et de reconstruction d'après-guerre, pour mettre l'accent sur le développement politique, institutionnel, économique et social, afin de favoriser un rapprochement avec l'Union européenne et permettre, en fin de compte, l'intégration au sein de l'UE. » ${ }^{11}$ En d'autres termes, on entend créer de futurs Etats membres. L'objectif de la transition apparaît dès lors clairement: rendre les pays de la région compatibles avec l'UE. Dans ces conditions, l'Union européenne est de toute évidence la principale instigatrice de leur transformation.

\section{L'adhésion à l'UE: moteur du progrès dans les Balkans occidentaux}

La perspective de l'adhésion à l'UE constitue, et de loin, la principale raison qui motive les pays des Balkans occidentaux et leurs populations à se soumettre au douloureux processus de la transition. En effet, la coopération - contestée au sein de l'opinion publique serbe et croate - avec le Tribunal pénal international de La Haye, l'application de l'accord-cadre d'Ohrid sur la paix et la réforme en Macédoine (Ohrid Framework Agreement), la réforme de la constitution en Bosnie et même la résolution pacifique du problème que pose le statut du Kosovo dépendent dans une large mesure de la crédibilité des promesses européennes. Ainsi, lorsque Tirana et Pristina adoptent une position modérée au sujet de la question albanaise, pour contenir les conflits potentiels, leur attitude découle directement de l'espoir d'adhérer à l'UE ${ }^{12}$. Cette perspective occupe la place d'alternative aux projets nationalistes qui préconisent la création d'Etat

10 Contrairement à la vision plutôt optimiste du Wiener Institut für Internationale Wirtschaft (wIIw), qui estime que la relance est alimentée non seulement par la consommation mais aussi par les investissements et les exportations, une étude du Secrétariat d'Etat à l'économie (seco) constate que l'argent transféré par les travailleurs émigrés vers leur patrie (dont l'Albanie, la Bosnie et la Serbie) exerce une influence plus grande que les investissements directs (cf. NZZ, 19. May 2006, p. 23, ou NZZ, 23. May 2006, p. 23).

11 «...from post-conflict rehabilitation and reconstruction towards political, institutional, economic and social development that should lead to rapprochement and ultimately integration into the European Union » (SDC, West Balkan Strategy 2007 to 2010, op. cit., p. 2 [traduction libre]).

12 Au Kosovo, en Macédoine, dans le sud de la Serbie et au Monténégro, la population albanaise est à peu près aussi nombreuse qu'en République d'Albanie. Les milieux politiques ne rêvent pourtant guère d'une «Grande Albanie» (qui réunirait tous les Albanais dans un seul pays). Même l'accordcadre d'Ohrid - qui est parvenu à éviter le conflit civil ayant failli opposer en 2001 l'armée gouvernementale à la guérilla albanaise et qui a étendu les droits de la minorité albanaise pour lui permettre de participer davantage à la gestion de l'Etat - a été «vendu» à la majorité macédonienne de la population comme une réforme pour se rapprocher de l'Europe. Sans cette perspective la population, réticente, l'aurait sans doute rejeté. 
nationaux ethniquement purs («Grande Serbie», «Grande Croatie», «Grande Albanie», etc.) pour sortir de l'oppression et de la misère. De telles formes d'Etat sont naturellement présentes dans la conscience collective de la région, mais seuls des groupes marginaux osent aujourd'hui les promouvoir activement. Il ne fait cependant aucun doute que l'option nationaliste refera surface si la perspective européenne tarde à se concrétiser. Le maintien durable de la paix sous l'égide de l'Europe repose donc essentiellement sur la mise en œuvre d'un calendrier approprié.

Le désir de mener une «vie normale», si souvent exprimé par la population, est étroitement lié à l'espoir d'adhérer à l'UE. Les gens lui associent une certaine prospérité, la levée des restrictions sur l'octroi de visas (qui les maintiennent prisonniers de leurs petits pays) et un accroissement de la sécurité individuelle. Lors des élections démocratiques et partout où le clientélisme ne parvient pas à fausser les votes, les électeurs jugent les élites politiques sur leur capacité à ancrer rapidement le pays dans le «port européen». Outre cette fonction pacificatrice, qu'il assure en suscitant aussi des attentes, le rapprochement entraîne de profonds changements institutionnels et sociaux. La procédure de stabilisation et d'association, qui transforme les Etats en candidats à l'adhésion, a engendré de véritables révolutions administratives, ne serait-ce qu'en obligeant les administrations à se doter des compétences nécessaires pour accueillir et répartir l'argent versé par Bruxelles. L'effort s'est le plus souvent traduit par des restructurations en profondeur ${ }^{13}$.

\section{La politique autonome de la Suisse dans une zone d'influence européenne}

La Suisse a réagi avec un certain retard à la stratégie de l'UE dans les Balkans occidentaux, mais a ensuite créé une section Europe du Sud-Est au sein de la DDC. Cette section a pour tâche de promouvoir le processus de transformation, l'intégration régionale et l'intégration des pays des Balkans occidentaux au sein de l'UE. Elle doit également intensifier les relations bilatérales avec les Etats de la région. Il est évident, et les textes l'affirment explicitement, que les activités suisses se réfèrent au processus de stabilisation et d'association (PSA) de l'UE et visent à y contribuer ${ }^{14}$. Au cours des années à venir (dès 2006), les moyens financiers consacrés à cet effort devraient se monter à 42 millions de francs environ.

13 Des critiques formulées au sein des pays concernés, mais aussi par des experts, prévoient cependant que les moyens mis à disposition pour créer des Etats membres ne sont de loin pas suffisants. Cf. Breaking out of the Balkan Ghetto: Why IPA Should Be Changed, rapport de 1'ESI, $1^{\text {er }}$ juin 2005, $<$ http://www.esiweb.org> (IPA: instrument d'aide de préadhésion). L'ESI, Initiative européenne pour la stabilité, est un institut de recherche et de politique fondé en 1999. Pour sa part, la Commission internationale sur les Balkans (International Commission on the Balkans), un groupe d'experts, de diplomates et d'anciens politiciens renommés d'Europe occidentale et des Balkans, a fustigé l'attitude toujours plus attentiste de 1'UE, l'accusant de manquer à sa parole. Alors qu'au sommet de Thessalonique, en 2003, les réformes internes constituaient la seule condition pour admettre les pays des Balkans occidentaux, l'UE insiste à présent sur sa propre capacité à intégrer de nouveaux membres en son sein. Pour la Commission sur les Balkans, la situation n'est guère réjouissante: «The future of Kosovo is undecided, the future of Macedonia uncertain, and the future of Serbia is unclear. We run the real risk of an explosion of Kosovo, an implosion of Serbia and new fractures in the foundations of Bosnia and Macedonia» (cf. ESI, Newsletter, n $^{\circ}$ 5, 2006, 10 May 2006, et les liens indiqués).

14 SDC, West Balkan Strategy 2007 to 2010, op. cit., p. 12 et suiv. 
Budget 2006 de la DDC pour les Balkans occidentaux (en millions de francs et en pourcentage)

\begin{tabular}{lcc}
\hline Pays & Mio de fr. & $\%$ \\
\hline Bosnie-Herzégovine & 8.0 & 19 \\
\hline Kosovo & 4.8 & 11 \\
\hline Serbie / Monténégro & 7.0 & 17 \\
\hline Albanie & 6.5 & 16 \\
\hline Macédoine & 5.5 & 13 \\
\hline Coopération régionale & 10.0 & 24 \\
\hline Total & 41,8 & 100 \\
\hline
\end{tabular}

Compétent dans ce domaine, le DFAE coordonne les activités de quatre intervenants: la DDC, responsable de la coopération technique; le seco, chargée de l'aide financière en tant qu'unité administrative du Département fédéral de l'économie (DFE); la Division politique I, qui entretient les relations diplomatiques; la Division politique IV, qui réalise des projets de promotion de la paix $^{15}$.

La Suisse pense pouvoir contribuer à la création d'Etats membres dans deux domaines principalement: la gouvernance (bonne gestion des affaires publiques) et le développement économique. Elle reprend à cet effet des projets en cours, réunis sous le titre «gouvernance», qui visent en particulier à renforcer les autorités politiques locales. Ce renforcement résulte d'un processus de décentralisation déjà en cours (Macédoine) ou en préparation (Kosovo et Serbie, par exemple) dans les pays de la région. Par ce biais, les projets souhaitent aussi stimuler la société civile, pour en faire un acteur dynamique et critique du changement. Comme le veut la coutume, les activités suisses réserveront une place particulière aux minorités. Pour ce qui est de l'économie, la Suisse entend améliorer le cadre légal dans lequel évoluent les entreprises, faciliter leur accès aux crédits et promouvoir la formation professionnelle. Outre la gouvernance et l'économie, le document stratégique définit quatre domaines (éducation, santé, eau et migrations) qui restent d'actualité dans certains pays. Enfin, les projets doivent accorder une grande attention aux relations hommesfemmes et à la jeunesse.

La promotion d'activités culturelles locales est un signe distinctif de la coopération suisse et les projets dans ce domaine sont réalisés par Pro Helvetia sur mandat de la DDC. Contrairement au British Council ou au Goethe Institut, la fondation Pro Helvetia ne représente pas la culture du pays donateur et ne cherche pas seulement à encourager les échanges culturels. Elle vise en effet à favoriser l'éclosion de paysages culturels locaux et régionaux dans les Balkans, car ces paysages sont perçus comme une composante essentielle de la société civile en gestation et d'une nouvelle identité culturelle capable de dépasser les cultures nationales ${ }^{16}$.

Il est judicieux de concentrer l'effort sur la construction d'Etats de droit démocratiques (l'accent étant certes mis sur la démocratie, mais davantage encore sur le droit), car cette concentration favorise l'intégration sociale, facilite

15 Ibid., p. 9.

16 Cf. Charles Landry, La culture au cœur du changement. Rôle de la culture dans le développement économique et social : enseignements du Programme culturel suisse, Berne, DDC; Pro Helvetia, 2006. 
l'émergence d'une société civile et crée des places économiques attrayantes. Voici comment: un Etat de droit fiable et efficace renferme un grand potentiel intégrateur en soi, car il garantit en principe au citoyen la possibilité de recourir à une instance neutre pour régler un conflit qui pourrait l'opposer à un autre citoyen ou aux services étatiques. Or, cette confiance à l'égard du système est presque totalement absente dans les Balkans occidentaux. Dès lors, les conflits se règlent en dehors du cadre légal, ou demeurent sans règlement. De plus, l'Etat de droit exerce une fonction particulière d'intégration dans les sociétés divisées et pluriethniques. En effet, les individus ne se sentent égaux que face à la justice (voire sur le marché économique), car les différences prédominent dans toutes les autres sphères sociales. On ne peut cependant pas espérer que les sociétés civiles des Balkans occidentaux, qui en sont à leurs balbutiements, parviendront - sans appui extérieur - à remplir dans un délai raisonnable la fonction d'une opinion publique pluraliste. Si un Etat de droit suffisamment fort ménage toutefois les espaces de liberté nécessaires, les citoyens finiront par s'y réunir (physiquement ou virtuellement) et à conclure des alliances pour défendre leurs intérêts. Dans les Balkans occidentaux, l'Etat de droit représente donc moins le résultat que la condition préalable, voire le catalyseur indispensable, à l'émergence d'une véritable société civile. La faiblesse notoire de l'autorité centrale dans tous les pays de la région, ainsi que sa polarisation politique et son manque d'efficacité, constituent probablement l'un des principaux obstacles au développement. Pour attirer des investissements durables, un pays doit commencer par garantir la sécurité du droit. Moyennant l'instauration des conditions requises, la relance économique viendra d'ellemême. La stratégie de la DDC semble toutefois indiquer que ni le marché économique ni la société civile ne suffisent à eux seuls pour assurer le développement. Les forces requises ne peuvent, à son avis, déployer toute leur créativité qu'en s'appuyant sur la sécurité et la liberté minimale garanties par un Etat de droit démocratique.

La politique suisse de développement sur place

Après cette approche théorique, considérons les avis émis dans les bureaux de coopération sur le développement des Balkans occidentaux. Il apparait d'emblée que la principale motivation de la politique suisse dans les Balkans, à savoir la prévention de la migration, se heurte à des contradictions. Les coordinateurs affirment presque d'une seule voix que les restrictions imposées par la Suisse à l'octroi de visas entravent le développement. Elles empêchent les étudiants, les universitaires et les hommes d'affaires de nouer des contacts, d'en tirer profit et de surmonter les cloisonnements dus à l'ethnocentrisme. Selon des sondages réalisés en Serbie, plus de $80 \%$ des étudiants ne sont encore jamais allés à l'étranger, le même constat valant sans doute pour d'autres pays de la région. Alors que certains responsables des bureaux de coopération plaident pour une suppression pure et simple du visa, d'autres préféreraient un système plus différencié. «Les criminels des Balkans, que nous n'avons bien entendu pas envie de laisser entrer en Suisse, ne font de toute façon pas la queue devant nos guichets pour obtenir un visa», déclarent plusieurs des personnes interrogées. Par ailleurs, nombre de gouvernements et d'organismes politiques demandent un 
système d'octroi de visas plus «intelligent» ${ }^{17}$. L'Union européenne, quant à elle, fait preuve d'une grande réserve et mise sur une meilleure sécurité des frontières et sur des pièces d'identité mieux protégées contre les faussaires. Le débat va sans doute se poursuivre et il est regrettable que le document stratégique de la DDC n'en parle qu'accessoirement ${ }^{18}$.

Par ailleurs, tous nos interlocuteurs ont souligné la nécessité de mieux harmoniser les activités des divers organismes d'entraide et organisations internationales. Mais cette volonté se heurte, elle aussi, à quelques obstacles: d'abord, on argue que l'harmonisation prend du temps et de l'énergie, et qu'elle atténue la visibilité ou masque l'origine suisse des projets. Dans les domaines les plus en vogue, telle la gouvernance locale, les divers donateurs se livrent concurrence pour attirer l'attention du public sur leurs projets. Par ailleurs, la coordination des activités n'est pas toujours du goût des bénéficiaires. «Sans elle, ils font trois voyages à l'étranger, au lieu d'un seul. Cela plaît à nombre d'entre eux.» Les responsables de la DDC saluent pourtant une harmonisation plus efficace des projets: «La coordination des activités dégage plus de ressources, nous donne plus d'influence et augmente l'efficacité du travail.» Elle assurerait en outre une meilleure répartition des risques.

Une autre tendance consiste à recourir davantage à des experts (consultants) de la région. On charge par exemple des spécialistes bulgares de créer des forums civiques en Serbie et en Macédoine pour stimuler la politique locale. Issus de la région, ces experts peuvent en général s'entretenir avec les partenaires du projet dans la langue maternelle de ces derniers, ils connaissent mieux les risques (et les potentiels) locaux et leur intervention coûte sensiblement moins cher que celle de leurs concurrents suisses ou ouest-européens. Cette tendance pourrait d'ailleurs se confirmer à l'avenir.

Les activités destinées à renforcer les autorités locales (gouvernance locale) visent tous les pays de la région et consistent à accroître les compétences des autorités municipales par le biais de la décentralisation. Cette réforme est particulièrement marquée en Macédoine, où l'application de l'accord-cadre d'Ohrid a amené le gouvernement à déléguer aux communes des pans entiers de la planification, ainsi que des systèmes éducatif et de la santé. Les partenaires considèrent souvent la décentralisation comme une compétence clé ou une spécialité de la Suisse, à tort selon les collaborateurs et les collaboratrices des bureaux de coopération. Certes, n'ayant jamais connu une structure centralisée, la Suisse n'a pas dû se décentraliser. Elle n'en possède pas moins une longue expérience de la démocratie et de la gestion communales, qui peut s'avérer utile pour les partenaires. Nul ne saurait nier, d'ailleurs, que la répartition du pouvoir entre plusieurs acteurs et niveaux est une spécialité suisse.

17 C'est l'avis exprimé par le ministre des Affaires étrangères de la Serbie-et-Monténégro, Vuk Draskovic, lors d'une conférence de presse tenue le 30 mai 2006 à Belgrade, conjointement avec le commissaire européen chargé de l'élargissement, Olli Rehn, et le coordinateur spécial du Pacte de stabilité, Erhard Busek. Compte tenu de la baisse des fonds que la diaspora envoie au Kosovo, certains experts craignent une détérioration de la crise sociale au Kosovo, au cas où l'Union européenne et la Suisse n'accorderaient pas au moins des permis de travail temporaires aux Kosovars (ESI, Cutting the Lifeline: Migration, Families and the Future of Kosovo, Berlin, Istanbul, 2006, p. II). Dans les conversations, les Kosovars déplorent souvent que la Suisse ait supprimé le statut de saisonnier.

18 «The Swiss migration policy will need to develop feasible strategies towards this challenging process », etc. SDC, West Balkan Strategy 2007 to 2010, op. cit., p. 5. 
L'une des questions qui reviennent sans cesse concerne une intégration plus efficace de la diaspora dans le processus de transformation. L'étude du seco mentionnée plus haut ${ }^{19}$ montre que les rapatriements de salaires, quoique très volumineux, ne sont guère investis de manière productive, car ils servent en majorité à acheter des biens de consommation et à construire des logements. Selon les estimations de tous les bureaux de coopération, leur impact sur l'économie est minime. Entre 2000 et 2001, la coopération au développement a tenté de créer un fonds d'investissement au Kosovo: la Suisse s'engageait à doubler les placements consentis par les émigrés. Le projet n'a jamais pris son essor, faute de confiance, voire de capitaux à investir sur un plus long terme. Mais le développement dépend avant tout du contexte politique et légal: si ce contexte est stable, les investissements arrivent automatiquement. Les acteurs économiques ont alors besoin de banques commerciales et non pas d'un fonds d'investissement géré par des organismes d'entraide. On songe apparemment moins à recourir au capital humain de la diaspora pour stimuler le développement. Les membres de la deuxième ou de la troisième génération, qui ont grandi et acquis une formation en Suisse, connaissent pourtant bien les conditions de vie dans les Balkans et pourraient contribuer à la modernisation. En Serbie, quelques entrepreneurs ont relevé le défi dans le domaine économique, mais l'accès aux sphères politiques semble plus difficile lorsque l'on revient de l'étranger. Là encore, d'aucuns ont toutefois réussi à se frayer un chemin: le mouvement de jeunesse Mjaft! en Albanie et quelques représentants de l'Union démocratique pour l'intégration, d'origine albanaise, en Macédoine.

Enfin, la coopération régionale s'intensifie aussi dans les activités de développement. Tous les responsables estiment que l'administration locale fait désormais l'objet d'une bonne coordination entre les bureaux de coopération, à tel point qu'ils envisagent de confier à l'un d'entre eux la responsabilité globale de certains projets d'envergure régionale. L'idée pourrait déboucher sur une matrice prévoyant la répartition des compétences entre les bureaux de coopération en fonction du mandat à exécuter, qui faciliterait ainsi le transfert de savoir-faire. En comparant les priorités des bureaux de coopération, on constate que trois domaines reviennent partout: la gouvernance, les infrastructures et la promotion de l'économie. Loin d'être un fruit du hasard, ce constat montre au contraire que la transition pose partout les mêmes problèmes. On peut dès lors se demander s'il ne serait pas judicieux de regrouper les projets de ces domaines au sein de programmes régionaux.

Outre les problèmes qui sous-tendent partout le processus de transformation, les pays sont également confrontés à des difficultés très spécifiques qui résultent de leur situation politico-ethnique. Quelle est la position de la politique suisse de développement dans ce domaine?

\section{La Macédoine}

En été 2001, la Macédoine a failli s'enfoncer dans une guerre civile interethnique et il a fallu les efforts concertés de la diplomatie de l'UE, des Etats-Unis et de l'Organisation du Traité de l'Atlantique Nord (OTAN) pour désamorcer le

19 Voir note 10. 
conflit. L'accord-cadre d'Ohrid (Ohrid Framework Agreement), conclu le 13 août 2001 sous la pression internationale, est à la fois un accord de paix et un accord de réforme destiné à renforcer la stabilité du pays. Outre l'interdiction du recours à la violence, il vise avant tout à décentraliser le pouvoir politique tout en préservant l'unité du pays. Grâce à des compétences élargies au niveau communal, la minorité albanaise bénéficie d'une plus grande autonomie dans les zones qu'elle habite. L'autonomisation favorise l'intégration nationale de cette minorité et améliore ses relations avec l'Etat macédonien. Le pays est candidat à l'adhésion à l'UE depuis fin 2005.

Dans le cadre du processus de décentralisation, le soutien suisse se concentre sur quatre domaines: la gouvernance, les infrastructures (eau, électricité et déchets), les services sociaux et le développement économique. La coopération s'intensifie aussi avec les services gouvernementaux, même dans des secteurs sensibles comme la coopération au sein du Partenariat pour la paix (un programme de l'OTAN). La Suisse propose ainsi des formations en communication et des cours sanitaires et sur les régions de montagne. Elle les organise parfois en collaboration avec les forces albanaises et croates, qui forment avec l'armée macédonienne le Groupe de l'Adriatique, désireux d'adhérer à l'OTAN. De plus, la création d'un poste d'attaché spécial devrait bientôt permettre d'améliorer la coopération sur le plan policier.

La Suisse œuvre également à l'élaboration d'une législation sur les langues, un domaine politique sensible où l'expérience de l'administration fédérale s'avère très utile. Une juriste suisse travaille au sein du bureau du vice-premier ministre, qui veille à l'application de l'accord d'Ohrid. Un programme de la Division politique IV s'articule autour du Processus de Mavrovo: des politiciens de tous les partis et de toutes les ethnies se réunissent régulièrement à Mavrovo, un lieu de villégiature renommé, et leurs entretiens servent à rétablir la confiance, tant au sein de la coalition gouvernementale interethnique qu'entre partis gouvernementaux et de l'opposition. Par ailleurs, des politiciens macédoniens et kosovars se retrouvent périodiquement à Skopje, dans le cadre d'une série de débats intitulée High Noon. Ces rencontres permettent de rendre les relations avec l'élite politique kosovare, naguère extrêmement tendues, bien plus constructives. Ces projets sont tous deux le fruit d'une initiative locale: les rencontres de High Noon ont été mises sur pied par un éditeur local, tandis que le Processus de Mavrovo a été organisé à la demande du gouvernement.

A Skopje, on souligne qu'il importe de pouvoir répondre rapidement aux nouveaux besoins, car le développement est en marche. Les responsables de la coopération suisse sont par ailleurs désireux de s'engager dans les domaines où l'Union européenne n'occupe pas la place de donatrice principale, à savoir la santé et la culture.

\section{La Serbie}

Depuis la chute de Slobodan Milosevic, à l'automne 2000, la transition connaît des hauts et des bas en Serbie. Des progrès rapides (telle la stabilisation macroéconomique) ont alterné avec des revers (assassinat du premier ministre Zoran Djindjic au printemps 2003). Après avoir pris un départ prometteur, les négociations en vue de la conclusion d'un accord de stabilisation et d'association avec 
l'UE ont été suspendues au printemps 2006, car Belgrade ne collaborait pas pleinement avec le Tribunal pénal de La Haye. L'UE lui reproche notamment de ne pas déployer beaucoup d'efforts pour arrêter et extrader Ratko Mladic, l'ancien commandant des Serbes de Bosnie, qui se trouverait toujours en Serbie.

La coopération suisse au développement (DDC, seco, DP IV) se concentre sur quatre domaines: l'administration locale, les infrastructures publiques (électricité), l'éducation et l'aide au secteur privé (promotion du commerce et des investissements et octroi de prêts aux PME par les banques locales, notamment).

Il s'avère plus aisé de collaborer avec les administrations locales plutôt qu'avec le gouvernement central. Les interlocuteurs se montrent non seulement plus coopératifs, mais changent aussi moins souvent. Pour ce qui est de la promotion des infrastructures publiques, la Suisse envisage de passer progressivement le relais aux banques. A l'avenir, le programme se concentrera davantage sur le sud de la Serbie, l'une des régions les plus pauvres du pays et à forte minorité albanaise, et sur la région du Sandzak, dans le sud-ouest, avec une forte population de Bosniaques (musulmans). On ne sait pas encore si le Monténégro, désormais indépendant, sera intégré dans ce programme et, si oui, comment. Le Kosovo possède son propre programme et le principal souci du bureau de coopération à Belgrade est de savoir si la solution qui sera apportée au problème du statut de la province engendrera un exode des Serbes du Kosovo vers leur «patrie». Les activités suisses visent à favoriser la réconciliation entre les vétérans des guerres et soutiennent des centres à Belgrade, à Sarajevo et à Zagreb, qui réunissent de la documentation sur les crimes de guerre.

\section{Le Kosovo}

Le Kosovo est aujourd'hui administré par l'ONU, mais son statut devrait bientôt être défini au niveau international. C'est dans cette province que l'on assiste véritablement à la création d'un Etat souverain. Dans bien des domaines, il a tout fallu recommencer à zéro. La Suisse est à l'œuvre dans quatre domaines: développement du secteur privé (agriculture, commercialisation, conseils et formation, crédits aux PME, etc.), infrastructures (adduction d'eau), gouvernance (administration, exécution des peines, cadastre et droits de la propriété) et développement social (formation professionnelle et réintégration des personnes déplacées).

Les besoins les plus urgents se situent du côté de l'Etat de droit et des capacités institutionnelles des autorités. Les organisations internationales présentes sur place et nombre d'organismes de développement se concentrent dès lors sur l'application de standards. Ceux-ci désignent les exigences minimales en matière de démocratie et de sécurité du droit, qui doivent garantir, en particulier mais pas uniquement, l'existence pacifique des minorités. Le travail sur ces standards doit être mené de front avec la résolution du statut du Kosovo. A l'heure actuelle, la solution la plus probable passe par une indépendance (conditionnelle) et le maintien d'une présence internationale réduite. Supposant qu'elles s'engagent pour une application plus efficace des standards, la Suisse apporte son appui aux femmes élues au Parlement. Elle soutient également la lutte contre le trafic d'êtres humains ainsi que des projets relevant de la justice transitoire (transitional justice). Parmi les grands problèmes, les col- 
laborateurs sur place évoquent une fixation sur la question du statut, à laquelle succombent les acteurs non seulement locaux, mais aussi internationaux. A leur avis, le nouveau statut du Kosovo ne va toutefois pas résoudre les problèmes du développement.

\section{La composante militaire de l'engagement suisse}

L'armée suisse a trouvé dans les Balkans un formidable terrain d'expérimentation, où elle teste de nouvelles formes d'engagement depuis le début des années 1990. Elle y a ainsi appliqué pour la première fois la doctrine de la sécurité par la coopération - parfois controversée en Suisse -, qui consiste à collaborer avec d'autres armées sans conclure d'alliance contraignante pour autant. La coordination des opérations avec le DFAE va de soi et a notamment été décrite dans un document commun, Papier stratégique pour l'engagement de la Suisse dans les opérations de maintien de la paix, daté du 5 juillet 2006. Les engagements de l'armée à l'étranger y sont définis comme une contribution complémentaire aux activités de la politique étrangère de la Suisse, et la «promotion militaire de la paix » comme une composante à part entière de sa politique extérieure et de sécurité $^{20}$. Le document souligne aussi que si la concentration de la promotion militaire de la paix sur les Balkans a été motivée par les conflits qui ont secoué la région, elle devra être réorientée à l'avenir. L'armée suisse pourrait par exemple s'engager dans des régions hors d'Europe, si cet engagement peut améliorer la sécurité de la Suisse. En conséquence, elle doit améliorer ses capacités pour participer à ce type d'opérations. A cet effet, elle ne devrait pas seulement augmenter le nombre de militaires mis à disposition (à 500 environ d'ici 2008), mais aussi se spécialiser dans la réalisation de «contributions de haute valeur»: recours à du matériel coûteux (hélicoptères par exemple) ou prestations très spécifiques dans les domaines du transport, de la police militaire, des renseignements, de l'information, de la collaboration entre les états-majors et de la formation. L'engagement de l'armée au Kosovo, par exemple, témoigne de la coordination étroite entre le DFAE et le DDPS dans la zone d'intervention «traditionnelle» que sont les Balkans ${ }^{21}$.

L'engagement de l'armée suisse au Kosovo a été décidé en été 1999, au lendemain de la guerre qui a opposé l'OTAN à l'ancienne République de Yougoslavie. En droit international, les opérations se fondent sur la résolution 1244 du Conseil de sécurité de l'onU. Adoptée le 10 juin 1999, celle-ci prévoit le déploiement d'une présence internationale de sécurité au Kosovo, dont la tâche principale est d'établir un «environnement sûr». C'est cette résolution qui a donné naissance à la KFOR (Kosovo Force), commandée par l'OTAN et réunissant aujourd'hui quelque 17'000 soldats. La Suisse met à sa disposition 220 mili-

20 DFAE, DDPS, Papier stratégique sur l'engagement de la Suisse dans des opérations de maintien de la paix, 5 juillet 2006, p. 2.

21 Si la mission au Kosovo représente, et de loin, le principal engagement de l'armée suisse à l'étranger, celle-ci participe aussi aux opérations de l'EUFOR (European Union Force) en Bosnie. La tâche des quelque 20 militaires sur place consiste à veiller à la sécurité et à assurer des transports en hélicoptère. Dans le cadre du Partenariat pour la paix (PPP) de l'OTAN, l'armée mène aussi des projets de formation en collaboration avec les armées albanaise et macédonienne (en particulier sur les régions de montagne et les services d'information). 
taires au maximum, qui servent au sein de la Swiss Company (swisscoy). Outre des tâches logistiques et des travaux de génie (construction de ponts surtout), les activités d'infanterie occupent une place croissante dans la mission: les soldats observent, contrôlent et patrouillent dans la région où ils sont stationnés et garantissent ainsi une protection aux minorités ethniques du Kosovo (Roms et Serbes principalement) ${ }^{22}$.

Le principal argument avancé pour justifier l'engagement de l'armée réside dans la sécurité intérieure «directement liée à la stabilité des Balkans, particulièrement au Kosovo ${ }^{23}$ : comme $10 \%$ environ des Albanais du Kosovo (soit quelque 200'000 personnes) vivent en Suisse, tout accroissement de la tension au Kosovo risque de radicaliser la diaspora, de provoquer des affrontements entre les Albanais du Kosovo et d' "autres colonies provenant de l'ex-Yougoslavie » ${ }^{24}$ (sous-entendu les Serbes vivant en Suisse) et d'engendrer de nouveaux afflux de réfugiés. «La Suisse a, plus que la plupart des pays européens, un intérêt national immédiat à un Kosovo pacifié, sûr et dont la population puisse avoir des perspectives d'avenir. ${ }^{25}$ Le Conseil fédéral souligne par ailleurs que la lutte contre la criminalité au Kosovo a des effets positifs sur la Suisse, qui serait «fortement touchée par des activités criminelles de groupes venant du Kosovo ${ }^{26}$. On retrouve ici à nouveau l'argument de «l'intérêt bien compris de la Suisse», avancé tant par le DDPS que par le DFAE pour justifier l'engagement de l'armée. Mais la création d'un environnement sûr et l'instauration d'un Etat à même d'exercer le pouvoir constituent sans nul doute les conditions préalables à un processus de transformation, qui fera du Kosovo un membre potentiel de l'UE et non pas un «Etat raté».

L'engagement financier est considérable: le rapport mentionné évalue les coûts annuels de la SWISSCOY à un peu moins de 34 millions de francs. A titre de comparaison, rappelons que l'aide au développement destinée à l'ensemble des Balkans occidentaux se monte à près de 42 millions de francs et que le Kosovo compte 2 millions d'habitants, alors qu'il y en a 20 millions dans l'ensemble de la région.

22 Après les violences perpétrées en mars 2004 contre des Serbes et des Roms du Kosovo, auxquelles la KFOR - surprise et mal équipée - a dû assister impuissante, la politique d'intervention et l'équipement des troupes ont été adaptés à la situation. L'accent est à présent mis sur la capacité de la KFOR à empêcher et à réprimer toute attaque violente contre des minorités (mais aussi contre la présence internationale et ses fonctionnaires). La mobilité des troupes a été accrue et elles sont désormais dotées d'un équipement antiémeutes (boucliers, matraques, gaz lacrymogène, fusils à balles en caoutchouc, etc.).

23 Conseil fédéral, Message sur l'arrêté fédéral concernant la participation de la Suisse à la Kosovo Force multinationale (KFOR) du 3 décembre 2004 (Feuille fédérale 2005 403), p. 404.

24 Ibid.

25 Ibid., p. 405.

26 Rapport 2005 sur l'engagement de la compagnie suisse (SWISSCOY) dans le cadre de la Kosovo Force multinationale (KFOR), p. 8. Mais il ne s'agit pas seulement de criminels. Le rapport souligne que, grâce à des relations étroites avec la Suisse, des personnes ayant par le passé obtenu la citoyenneté suisse - autrement dit des Suisses - occuperont à l'avenir des postes en vue, tant en politique que dans le monde des affaires (p. 7). 
Alors que la coordination des prestations d'aide et de développement des nombreux intervenants de la communauté internationale pose de toute évidence un problème (voir ci-dessus), la cohérence théorique et pratique entre les divers acteurs suisses de la coopération est largement acquise. Toutes leurs activités s'inscrivent dans les quatre grands domaines suivants: instauration de la paix, stabilisation politique, transition économique et création d'Etats membres.

L'engagement de la Suisse dans les Balkans occidentaux se distingue donc par une grande cohérence. Dans ce contexte, la déclaration sur le statut du Kosovo, faite par le DFAE à l'ONU, a de quoi surprendre et paraît peu réfléchie, car elle est en contradiction avec les activités de la Division politique IV, des bureaux de coopération sur place et des ambassadeurs suisses dans la région.

Le 27 mai 2005, le représentant helvétique au siège de l'onU à New York a déclaré que la Suisse pense qu'il convient d'accorder une «indépendance formelle» au protectorat du Kosovo. La déclaration soutient que c'est la seule solution réaliste si l'on considère à la fois le désir légitime des minorités de vivre en sécurité et le désir légitime de la majorité de s'autodéterminer. L'application de la solution devrait faire l'objet d'un suivi international et comprendre une décentralisation du territoire, pour permettre aux minorités de prendre une part plus active aux processus politiques. Bien que cette proposition insiste bien sur les diverses conditions préalables à une accession à l'indépendance, les deux parties n'ont retenu que le mot «indépendance». Les réactions ne se sont pas fait attendre: euphorie du côté des Albanais du Kosovo, refus net de la part des Serbes.

Les projets menés notamment par la Division politique IV pour promouvoir le dialogue entre les deux parties (Processus de Lucerne) sont tombés à l'eau: les Serbes ont refusé d'y participer, jugeant les organisateurs trop partiaux. Un travail préparatoire de plusieurs années a été anéanti d'un simple trait.

Puisque la politique extérieure, à plus forte raison celle menée dans les Balkans, sert à défendre nos intérêts, quels sont les intérêts défendus par la prise de position de la Suisse? Dans un article publié dans le quotidien Der Bund, Micheline Calmy-Rey, cheffe du DFAE, a motivé la déclaration suisse par la présence, dans notre pays, d'une importante diaspora, kosovare notamment. L'argument étonne. En effet, vu la présence des diasporas serbe et kosovare, la Suisse aurait plutôt dû s'en tenir à une stricte neutralité. Celle-ci lui aurait permis de faire valoir ses bons offices et de rétablir le dialogue, pour le mener aussi bien avant que pendant et après la résolution de la question du statut du Kosovo. Dans une pirouette étrange, la conseillère fédérale souligne le courage de la Suisse, qui a osé parler ouvertement d'indépendance, alors que les autres Etats se taisaient encore $^{27}$. La déclaration témoignait plus de l'empressement à exprimer le fond de sa pensée qu'elle ne formulait une politique devant servir l'intérêt de la Suisse à voir le différend trouver une solution pacifique et aussi consensuelle que possible. Citant la conseillère fédérale, le quotidien Koha Ditore de Pristina 
écrit que le gouvernement (suisse) ne serait pas allé trop vite en besogne en suggérant l'indépendance. «Nous voulions susciter des réactions. Il faut parfois que quelqu'un ose dire tout haut ce que tout le monde pense tout bas. $»^{28}$

En réalité, tout donne à penser que les travaux du groupe de contact qui mène les négociations entre Belgrade et Pristina s'acheminent vers une indépendance conditionnelle du Kosovo. Et il existe de bonnes raisons de croire que c'est une solution réaliste. Mais là n'est pas la question. En effet, il faut plutôt se demander s'il est bon que la politique suisse dans les Balkans prenne parti dans le dernier des conflits passionnés qui agitent la région. Cette attitude lui permet-elle d'élargir sa marge de manœuvre? C'est loin d'être le cas, bien au contraire. La Suisse ne joue aucun rôle sur le grand échiquier international et elle s'est à présent aussi fermé les niches qu'elle aurait pu occuper en œuvrant pour le dialogue et la réconciliation entre Serbes et Albanais. Les entretiens avec des conseillers de la cheffe du DFAE n'ont pas permis de cerner l'avantage politique de cette prise de position unilatérale, ni à court terme ni à long terme ${ }^{29}$.

Dans ces conditions, deux éléments sont particulièrement déconcertants. Premièrement, les diplomates et les experts des ambassades et des bureaux de coopération sur place n'ont pas été consultés auparavant, mais placés devant le fait accompli. Les Commissions de politique extérieure du Conseil national et du Conseil des Etats se sont d'ailleurs trouvées dans la même situation. Deuxièmement, il est surprenant qu'un tel revirement dans un domaine prioritaire de la politique étrangère suisse n'ait pas fait l'objet d'un arrêté du Conseil fédéral, mais ait été décidé à la tête du DFAE. Tous les conseillers fédéraux ont en effet été informés par la presse. Certes, il ne faut pas accorder trop d'importance à cette déclaration, puisqu'elle est passée largement inaperçue au sein de la communauté internationale. Mais cette manière de prendre fait et cause pour une partie en présence discrédite les activités que la Suisse mène depuis de longues années, souvent avec un succès remarquable et une force de conviction rare lorsqu'il s'agit de conjuguer ses propres intérêts avec ceux de la population des Balkans.

28 Cité dans le OSZE-Pressemonitor de Pristina, 27 juillet 2005.

29 Un de nos interlocuteurs albanais en Macédoine (ambassadeur dans un pays de l'UE) interprète à sa manière cette manœuvre surprenante: si la Suisse souhaite assister dès que possible à la création d'un Etat indépendant du Kosovo, c'est pour pouvoir se débarrasser des Albanais du Kosovo qu'elle ne veut plus voir chez elle. 